DOCUMENTS

pour I'histoire
des techniques
Documents pour l'histoire des techniques

Nouvelle série

$15 \mid 1^{\text {er }}$ semestre 2008

Minorités et circulations techniques du Moyen-Âge à l'époque Moderne

Bernard Bacher, Jean-François Brun, Éric Perrin, La manufacture d'armes de Saint-Étienne : la révolution des machines, 1850-1870

Saint-Étienne, Musée d'art et d'industrie, 2007, 183 pages.

François Jarrige

\title{
OpenEdition
}

Journals

Édition électronique

URL : http://journals.openedition.org/dht/1075

DOI : $10.4000 /$ dht. 1075

ISSN : 1775-4194

Éditeur :

Centre d'histoire des techniques et de l'environnement du Cnam (CDHTE-Cnam), Société des élèves du CDHTE-Cnam

Édition imprimée

Date de publication : 1 juin 2008

Pagination : 233-235

ISBN : 978-2-95-30779-1-9

ISSN : 0417-8726

Référence électronique

François Jarrige, « Bernard Bacher, Jean-François Brun, Éric Perrin, La manufacture d'armes de SaintÉtienne : la révolution des machines, 1850-1870 ", Documents pour l'histoire des techniques [En ligne], 15। $1^{\text {er }}$ semestre 2008, mis en ligne le 22 octobre 2010, consulté le 22 septembre 2020. URL : http:// journals.openedition.org/dht/1075; DOI : https://doi.org/10.4000/dht.1075

Ce document a été généré automatiquement le 22 septembre 2020

(c) Tous droits réservés 


\section{Bernard Bacher, Jean-François Brun, Éric Perrin, La manufacture d'armes de Saint-Étienne : la révolution des machines, 1850-1870}

Saint-Étienne, Musée d'art et d'industrie, 2007, 183 pages.

François Jarrige

\section{RÉFÉRENCE}

Bernard Bacher, Jean-François Brun, Éric Perrin, La manufacture d'armes de Saint-Étienne : la révolution des machines, 1850-1870, Saint-Étienne, Musée d'art et d'industrie, 2007, 183 pages.

En 2001, la production d'armes s'arrêtait à Saint-Étienne après plusieurs siècles d'activité dynamique. À l'image de nombreuses autres régions industrielles en phase de reconversion, la valorisation de la mémoire du patrimoine industriel et technique local devient un enjeu primordial pour le futur développement du bassin stéphanois. C'est dans cette préoccupation que s'enracine la publication de cet ouvrage initié par le musée d'art et d'industrie de Saint-Étienne. Il marque à la fois l'aboutissement d'une enquête collective menée par une équipe d'historiens des techniques et de l'industrie passionnés par le patrimoine local et la conclusion d'une exposition qui s'est tenue dans la ville en 2007. Ni une simple monographie érudite sur la production d'armes de guerre à Saint-Étienne, ni un catalogue d'exposition déconnecté des préoccupations historiennes, l'ouvrage permet une passionnante plongée dans le quotidien de la production d'armes durant la première industrialisation. Il décrit avec minutie ce que les auteurs appellent la "révolution des machines ", c'est-à-dire le passage d'une production artisanale dans des petits ateliers dispersés selon le modèle du Verlagssystem (même si les auteurs n'emploient pas cette notion de l'historiographie 
économique) à une production mécanisée et concentrée dans une vaste manufacture édifiée sous le second Empire.

2 Le premier chapitre est consacré aux "héritages historiques » : il aborde l'action de l'État de l'époque moderne pour stimuler les productions d'armes, les premières tentatives de mécanisation sans lendemain tentées à la veille de la Révolution ${ }^{1}$, le processus de concentration durant la première moitié du XIX ${ }^{\mathrm{e}}$ siècle. Mais avant le second Empire, l'organisation du travail reste traditionnelle. La main-d'œuvre est dispersée dans de petits ateliers, l'usage des outils et la fierté du savoir-faire priment sur la mécanisation à laquelle les artisans s'opposent périodiquement. Les auteurs mentionnent en effet les révoltes et les conflits récurrents que suscitent les tentatives de mécanisation du travail, que ce soit dans les années 1780, en 1831 ou, d'une façon plus souterraine, dans les années 1860. Toutefois, à partir de cette date, une réorganisation complète de la production et $\mathrm{du}$ travail intervient et relègue définitivement « l'homme habile au rang de servant de la machine "infaillible" » (p. 42). L'essentiel de l'ouvrage concerne la période du second Empire qui voit la réalisation du programme de mécanisation tant rêvé.

3 Dans le deuxième chapitre, les auteurs décrivent les conceptions novatrices et les contraintes qui gouvernement la mécanisation. Durant cette période, les conflits de grande ampleur comme la guerre de Crimée ou la guerre de Sécession aux États-Unis poussent à l'évolution des tactiques militaires, ce qui accentue les exigences à l'égard de la production d'armes. Le site de Saint-Étienne, qui bénéficie à la fois de la présence du charbon et d'un secteur métallurgique dynamique, s'impose comme le principal pôle français de l'arme militaire individuelle. Frédéric-Guillaume Kreutzberger, un Français qui avait travaillé aux États-Unis, joue un rôle décisif dans l'importation de nouvelles méthodes permettant l'interchangeabilité des pièces. Il offre ses services à Napoléon III en vue de mécaniser le travail existant et de réorganiser la production. Le perçage du canon et l'usinage extérieur sont les opérations qui sont mécanisées les plus précocement et les plus efficacement. Les canons en fer étaient traditionnellement usinés au moyen de meules et de limes, puis polis manuellement. Mais l'apparition de l'acier modifie sensiblement l'approche de l'usinage: la résistance croissante des métaux autorise la mise au point d'outils et de machines performantes remplaçant avantageusement le travail manuel. Toutes les étapes du travail ne sont toutefois pas aussi aisément mécanisables, le garnissage du canon (c'est-à-dire les opérations de finition) et le rayage, en particulier, restent plus difficiles à automatiser.

Dans la troisième partie, les auteurs examinent les étapes de la construction et de l'installation de la nouvelle manufacture dans les années 1860, après l'étude des principes généraux ils examinent les étapes de leur réalisation. Les conceptions technologiques et organisationnelles de Kreutzberger jouent un grand rôle dans la conception de l'espace de production. L'exigence d'efficacité est au cœur du projet : on cherche les solutions les plus économes dans le déplacement des pièces et dans la disposition des hommes et des machines. Le schéma d'organisation imaginé par Kreutzberger est retenu en 1863-1864. Dans la nouvelle manufacture stéphanoise, les machines-outils doivent être mues par une force motrice importante et régulière, d'où le recours exclusif à la vapeur. Les auteurs examinent avec finesse la disposition des machines à vapeur, les souterrains creusés pour installer les arbres de transmission, l'organisation savante de l'espace de production. Une fois les principes fixés, il s'agit de procéder à l'implantation de l'usine. En 1863, un accord est signé entre la mairie de 
Saint-Étienne et le ministère de la Guerre : la mairie est chargée d'acquérir les terrains (grâce à des décrets d'expropriation pour cause d'utilité publique) et de réaliser divers aménagements et travaux de viabilisation du site retenu. En 1864, les travaux démarrent selon une logique qui révèle toujours la priorité accordée à la production : on commence par édifier « la grande usine » qui doit accueillir les machines et toute l'organisation est conçue pour éviter au maximum de perturber la production. En avril 1866, la nouvelle manufacture est inaugurée. Dès la fin de l'année 1867, les principales constructions sont achevées, d'intéressantes photos datées de 1866 et 1867 (p. 125, 128-129) montrent d'ailleurs l'avancée des travaux et la physionomie de ce vaste chantier.

5 En 1866, le gouvernement de Napoléon III avait adopté officiellement le fusil modèle Chassepot (canon en acier, culasse mobile et percussion à aiguille) comme arme réglementaire, il avait décidé d'en faire fabriquer 1,2 millions en quatre ans. En 1870, ce programme était presque réalisé, puisque un million avaient été fabriqués, dont $50 \%$ provenaient de Saint-Étienne. Même si cet effort d'armement n'a pas suffi à assurer la victoire contre la Prusse en 1870, la « Révolution des mécaniques » a bien eu lieu à Saint Étienne dans la deuxième moitié des années 1860 . Comme le note les auteurs en citant l'ingénieur Kreutzberger, la révolution des mécaniques devait aussi être une révolution dans les esprits en face $d$ '« un personnel d'ouvriers qui ne considère le concours du travail des machines que comme un ennemi implacable » (p. 95). La mécanisation en effet est autant un phénomène social que technique, elle nécessite de redéfinir les normes qui gouvernent les ateliers, de repenser les stratégies de gestions de la maind'œuvre. On aurait aimé que ces aspects soient davantage développés, ce qui aurait permis de mieux comprendre les réactions de la main-d'œuvre à l'égard de la mécanisation. Les auteurs s'attachent finalement peu aux gestes du travail, ils mentionnent des résistances mais sans réellement questionner leurs significations, se contentant parfois de reproduire les jugements des contemporains sur le caractère naturellement routinier de la main-d'œuvre qu'il faut éduquer. Mais l'étude de l'évolution de la main-d'œuvre locale, de ses réactions et de son organisation aurait été une autre histoire.

6 En définitive, l'ouvrage richement illustré par des photos d'époque, des schémas de machines, des plans d'usines, des reproductions de documents issues des archives, s'avère d'une lecture agréable et d'un grand intérêt. Des encarts éclairent utilement la biographie de certains personnages, des annexes substantielles fournissent de nombreux éléments pour de futures recherches. Tout cela fait de cet ouvrage un apport important à l'histoire de l'industrie, des techniques et du travail au XIX ${ }^{e}$ siècle. Au-delà $\mathrm{du}$ cas, en lui-même intéressant, du bassin stéphanois, il éclaire quelques zones d'ombre de la genèse de la société industrielle et technologique contemporaine. 


\section{NOTES}

1. Signalons la publication récente d'un article riche sur les efforts de mécanisation de l'industrie des armes à Saint-Etienne à la fin du XVIII ${ }^{\mathrm{e}}$ siècle qui complète utilement cet ouvrage : J. L. Viret, «L'industrie des armes portatives à Saint-Etienne, 1777-1810. L'inévitable mécanisation? », Revue d'histoire moderne et contemporaine, 54-1, janvier-mars 2007, pp. 171-192.

\section{AUTEURS}

\section{FRANÇOIS JARRIGE}

Centre de recherches en histoire du XIXe siècle, Université Paris-I 